\title{
Non-data Aided Timing Phase Recovery Scheme for Digital Equalization of Chromatic Dispersion and Polarization Mode Dispersion
}

\author{
Jangwoo Park and Wonzoo Chung* \\ Division of Computer and Communication Engineering, Korea University, Seoul 136-701, Korea \\ Jongsun Park \\ School of Electrical Engineering, Korea University, Seoul 136-701, Korea \\ Sungchul Kim \\ Department of Communication Engineering, Myongji University, Yongin 448-728, Korea
}

(Received July 6, 2009 : revised August 4, 2009 : accepted August 4, 2009)

\begin{abstract}
In this paper we propose an electronic domain timing phase selection scheme for the optical communication systems suffering from inter-symbol-interference (ISI) distortion due to chromatic dispersion (CD) or polarization mode dispersion (PMD). In the presence of CD/PMD a proper timing phase selection is important for discrete time domain equalizers, since different timing phases produce different nonlinear ISI channels of different severity. The proposed timing phase recovery scheme based on dispersion minimization (DM) practically approximates the optimal minimum mean squared error (MMSE) timing phase without training signals which reduces overall throughput substantially, especially in time-varying channels such as PMD. The simulation results show that the proposed DM timing agrees with MMSE timing phase, under proper normalization of the received signals, for various dispersion and OSNR.
\end{abstract}

Keywords: Chromatic dispersion, Polarization mode dispersion, Inter symbol interference equalization, Timing phase

OCIS codes: (060.2360) Fiber optics links and subsystems; (060.4510) Optical communications

\section{INTRODUCTION}

To meet the ever increasing demands of high data rate, optical fiber communication systems now need to deal with inter-symbol interference (ISI) caused by chromatic dispersion $(\mathrm{CD})$ and polarization-mode dispersion (PMD) [1-3]. The electrical domain dispersion compensation techniques for $10 \mathrm{~Gb} / \mathrm{s}$ or beyond have been intensively studied recently [4-8]. There have been largely three approaches to mitigate ISI in optical communication systems; conventional linear equalizers and decision feedback equalizers (DFEs) [4-5], maximum likelihood sequence estimation (MLSE) equalizers [6], and nonlinear DFEs based on Volterra theory [7, 8]. The MLSE equalizer is optimal, but requires perfect ISI

*Corresponding author: wchung@korea.ac.kr channel estimation and noise statistics. Furthermore, its complexity increases exponentially as the channel length increases. On the other hand, linear or nonlinear equalization scheme is sub-optimal, but it is compensated by its computational efficiency and simplicity in that it does not directly require channel estimation and noise statistics.

However, these electronic domain dispersion mitigation studies have been focused on the equalization schemes only, and the importance of timing phase for equalizers has not gained much attention so far $[9,10]$. In fact, it has been well known in the wireless communication community that depending on the timing phase the resulting discrete time domain inter-symbol interference (ISI) can be varied [11, 12]. Furthermore, some timing phases produce better ISI channels to deal with equalizers. The optimal timing phase would be the one 
that produces the mildest ISI channel for the equalizer to mitigate. Conventionally the timing phase with minimum mean squared error (MMSE) between the sampled received signal and the originally sent data is the optimal one to bring the desired ISI channel. However, this MMSE timing phase requires a training signal. In the absence of dispersion distortion, the selection of the MMSE timing phase is equivalent to adjusting the delay and can be set infrequently and remain fixed. However, in the presence of PMD distortion the optimal timing phase varies over time and adjusting the MMSE timing phase with training signals is wasting valuable bandwidth. A non-data aided timing phase recovery scheme is desired for the optical communication system.

In this paper, we replace the MMSE criterion with dispersion minimization (DM) criterion to blindly determine the optimal timing phase. DM criterion is based on the fourth order statistics of the received data and is a commonly used approach in wireless communication systems to replace MMSE criterion [12]. DM timing phase can be implemented by block by block approach or adaptive algorithm using a stochastic update equation. Simulation results for On-Off keying (OOK) systems provided in section III shows that DM timing phase agrees with MMSE timing phase for several $\mathrm{CD} / \mathrm{PMD}$ impaired channels and optical signal to noise ratio (OSNR).

\section{OPTIMAL TIMING PHASES FOR ELECTRONIC DOMAIN EQUALIZERS}

\section{Timing phase and ISI channel}

Consider a typical non-return-to-zero on-off-keyed (NRZ-OOK) optical communication model in Fig. 1. The bit sequence $\left\{b_{k}\right\}$ with a bit rate $T$ is pulse shaped by a continuous time pulse $p(t)$ (for example, a raised cosine pulse with roll-off factor 1),

$$
s(t)=\sum_{k=-\infty}^{\infty} b_{k} p(t-k T)
$$

and transmitted over an optical fiber after laser modulation. We assume the fiber is a lossless linear channel with $\mathrm{CD}$ and first order PMD distortion. The CD and
PMD are generally non-linear distortions, but often modeled as a linear distortion by a first order approximation in the optical field domain. The effect of dispersion can be modeled as a linear filtering process given by

$$
r_{o}(t)=h_{o}(t) \otimes s_{o}(t)
$$

where $S_{o}(t), r_{o}(t)$, and $h_{o}(t)$ are the transmitted, received signals, and the fiber impulse response in optical field domain, respectively, and $\otimes$ denotes convolution. The first-order PMD can be characterized using the principal states model [3], where the fiber is described as a one-input two-output device, with each output corresponding to an output principal state of polarization. In the frequency domain, the impulse function describing the first order PMD is given by

$$
H_{o}(f)=\left[\begin{array}{c}
\sqrt{\gamma} \exp \left[j 2 \pi f\left(\frac{-\tau}{2}\right)\right] \\
\sqrt{1-\gamma} \exp \left[j 2 \pi f\left(\frac{\tau}{2}\right)\right]
\end{array}\right]
$$

where $\gamma$ is the power splitting ratio, informing how the power of the input light of the fiber is divided onto the two input principle state of polarization, and $\tau$ denotes the differential group delay between the two polarizations. Incorporating CD distortion, the fiber transfer function becomes [5]

$$
H_{o}(f)=\left[\begin{array}{c}
\sqrt{\gamma} \exp \left[j 2 \pi f\left(\frac{-\tau}{2}\right)+\frac{j D L(2 \pi f)^{2} \lambda^{2}}{4 \pi c}\right] \\
\sqrt{1-\gamma} \exp \left[j 2 \pi f\left(\frac{\tau}{2}\right)+\frac{j D L(2 \pi f)^{2} \lambda^{2}}{4 \pi c}\right]
\end{array}\right]
$$

where $L$ denotes the fiber length, $\lambda$ the wavelength, $D$ the dispersion parameter at $\lambda$, and $c$ the speed of light.

The received optical signals suffered from these dispersions are demodulated to electronic signal by a square-law detector. In contrast to the signal detection process in wireless communication systems, this process is nonlinear. Hence, the linear convolution relation in the optical field domain is not preserved in the

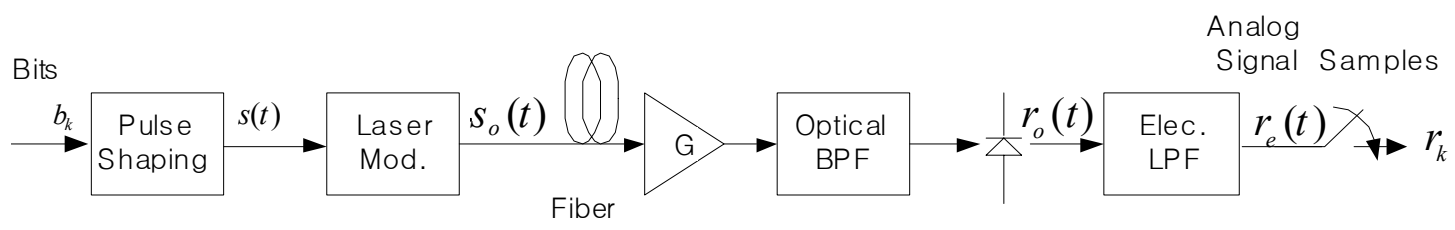

FIG. 1. Fiber communication system using NRZ-OOK. 
baseband electronic domain. The received signal in the electronic domain is

$$
r_{e}(t)=\left|h_{o}(t) \otimes s_{o}(t)+w_{o}(t)\right|^{2}+w_{e}(t)
$$

where $w_{o}(t)$ denotes noise in the optical domain due to fiber amplifier and $w_{e}(t)$ denotes the noise in the electronic domain. In discrete time domain, by denoting $r_{k}:=r_{e}(k T+\tau)$ with respect to a timing phase $\tau$,

$$
r_{k}=\left|\sum_{n-\infty}^{\infty} c_{o}((n-k) T+\tau) b_{k}+w_{o}(k t+\tau)\right|^{2}+w_{e}(k T)
$$

where $c_{o}(t)$ denotes the combined impulse response with the pulse shaping filter $p(t)$ and $\mathrm{PMD} / \mathrm{CD} h_{o}(t)$. We assume that the amplified-spontaneous-emission (ASE) noise from the erbium-doped fiber amplifiers dominates and the electronic domain noise is ignorable. Then

$$
\begin{aligned}
r_{k} & =\sum_{n}^{\infty}\left|c_{0}((n-k) T+\tau)\right|^{2} b_{n}^{2}+2 \sum_{n \neq m}^{\infty} \operatorname{Re}\left(c_{0}((n-k) T+\tau) c_{o}^{*}((m-k) T+\tau)\right) b_{n} b_{m} \\
& +2 \operatorname{Re}\left(\sum_{n} c_{0}((n-k) T+\tau) b_{n} w_{o}^{*}(k t+\tau)\right)+\left|w_{o}(k t+\tau)\right|^{2}
\end{aligned}
$$

In the absence of dispersion, the channel impulse response, $C_{0}$, satisfies Nyquist criterion [11], i.e., there exist an optimal timing phase $\tau$ such that

$$
c_{o}(k T+\tau)= \begin{cases}1 & k=0 \\ 0 & k \neq 0\end{cases}
$$

Hence, we can successfully recover the input $b_{k}$. However, in the presence of dispersion, there does not exist such a timing phase $\tau$, and, consequently, the optimal timing phase would be the one resulting in the mildest dispersion. Notice that from (7), the linear component of ISI sufficiently represents the ISI distortions due to dispersion.

Figure 2 shows the different ISI distortions represented by the linear portion resulting from different timing phases for CD/PMD channel for $L=80 \mathrm{~km}$ SMF with the CD parameter $D=10 / \mathrm{ps} / \mathrm{km} / \mathrm{nm}$ and $\Delta \tau=60 \mathrm{psec}$ differential group delay. Figure 2-a) shows three different timing phases $\left(\tau_{A}, \tau_{B}, \tau_{C}\right)$ and Figure 2-b) shows corresponding linear ISI component in baud space (each tap corresponds to 0.1 ns delay). Among three timing phases $\tau_{B}$ produces the mildest ISI channel with suppressed pre-cursors and post-cursors, while other timing phases produces ISI channels with large precursor and post cursor. This example illustrates the importance of the timing phase.

The optimal timing phase in the mean squared error (MSE) sense can be defined as

$$
\tau_{M M S E}=\arg \min _{\tau} E\left(r_{k}(\tau)-b_{k}\right)^{2}
$$

where we assume that the sampled signal is normalized by

$$
r_{k}(\tau)=\frac{r_{k}(\tau)}{E\left(r_{k}(\tau)-E\left(r_{k}(\tau)\right)^{2}\right.}
$$

(In the example in Figure 2, $\tau_{B}$ is the MMSE timing phase.) In order to find the optimal MMSE timing phase, a training signal is required. However, the ISI channel is often time-varying, as PMD has time-varying nature, and frequent training signals to obtain the optimal timing phase are not available. Hence, a nondata-aided optimal timing phase scheme is desired.

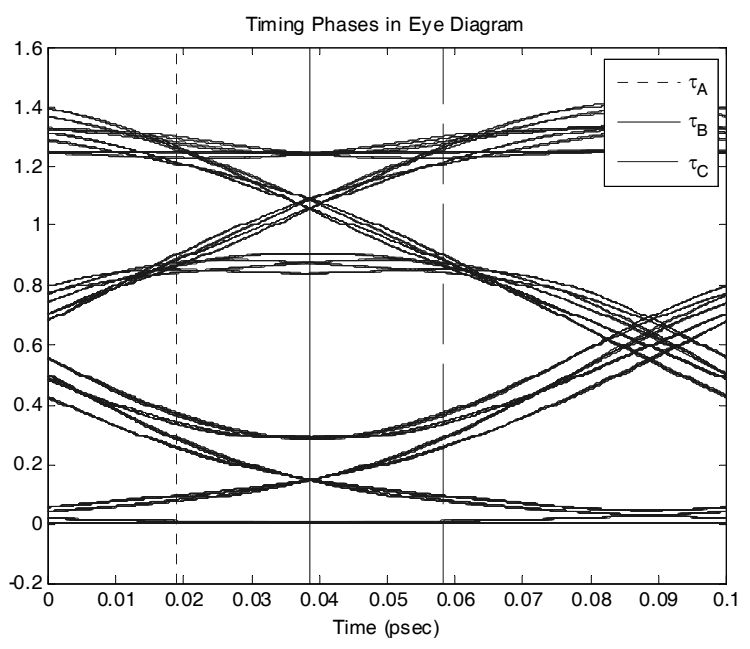

(a) Timing phases in eye diagram
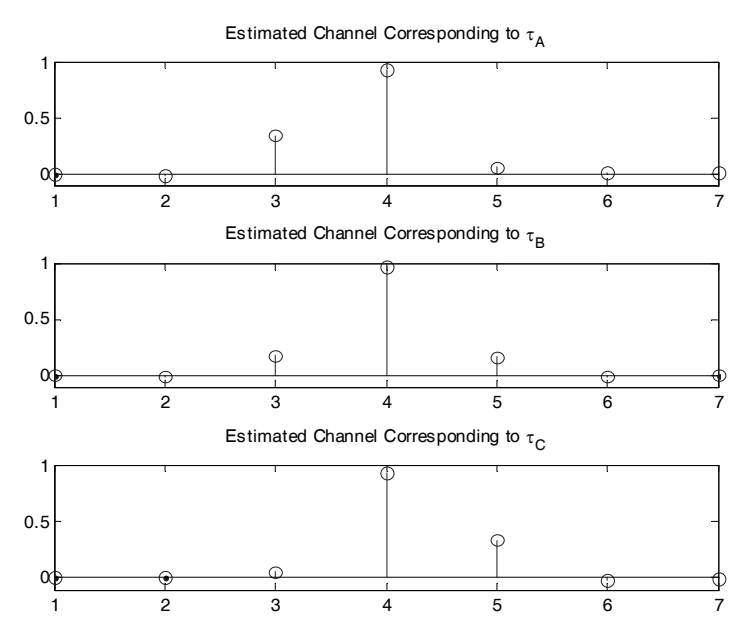

(b) Estimated linear ISI channel

FIG. 2. ISI distortion due to timing phase. 


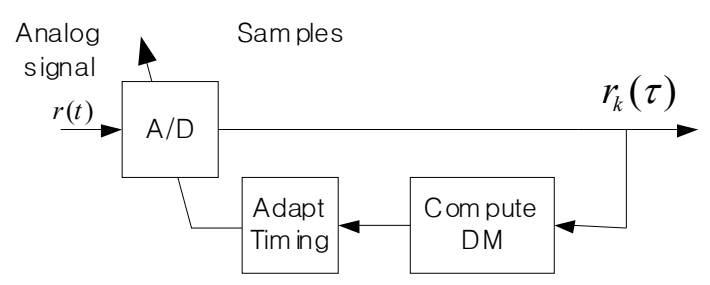

FIG. 3. DM timing recovery loop.

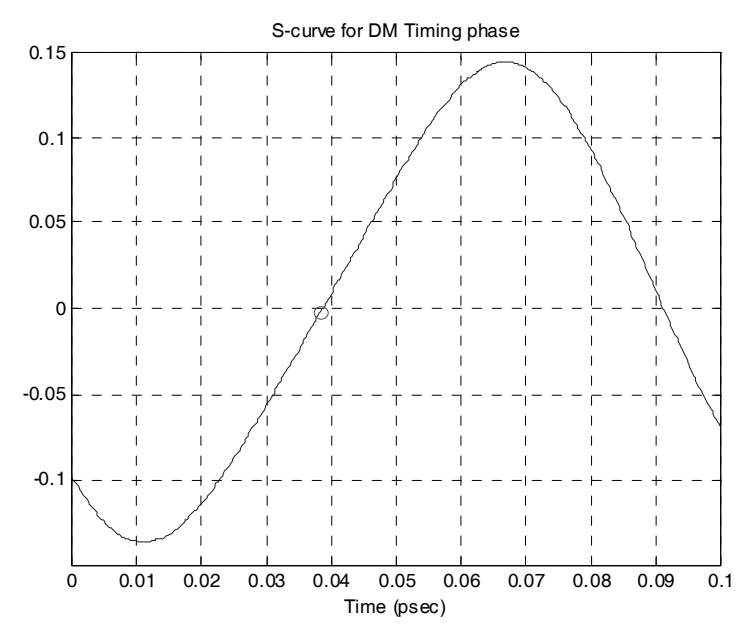

FIG. 4. S-curve for adaptive DM timing algorithm.

\section{Dispersion Minimization Timing Phase Criterion}

In digital communication, when a training signal is unavailable, MSE criterion is often replaced by dispersion minimization (DM) criterion [12,13]. The intuition behind of DM criterion is that the transmitted digital data have only certain finite discrete values (for example $\{ \pm 1\}$ for BPSK signaling) and by minimizing the dispersion of the received signal one could remove the adversary effects due to the distortion. The optimal timing in DM sense is given as

$$
\tau_{D M}=\arg \min _{\tau} E\left(\left|r_{k}(\tau)\right|^{2}-\gamma\right)^{2}
$$

where $\gamma$ is a constant, known as the dispersion constant [12], which is set to 0.5 for OOK and 1 for DPSK. Notice that the DM cost is similar to the MSE cost except that the training signal is replaced by the dispersion constant and the received signal is squared to remove phase sensitivity. The extra benefit of the DM approach is its simplicity; it requires almost the same complexity as the training based LMS approach. Furthermore, it can be implemented via a stochastic adaptive algorithm: We can use an adaptive approach to find $\tau_{D M}$ by the following stochastic gradient update equation [14]

$$
\tau(k+1)=\tau(k)+\mu\left(\left|r_{k}(\tau(k))\right|^{2}-\gamma \mid\right)\left(r _ { k } \left(\tau(k)-r_{k}(\tau(k-1))\right.\right.
$$

A timing phase recovery system using this stochastic equation can be implemented as the block diagram depicted in Figure 3.

Figure 4 shows the S-curve for this DM adaptive algorithm for the example in Figure 2, which indicate the convergence behavior of the adaptive algorithm [11]. The zero-crossing occurs at the MMSE timing phase, which indicates that the DM timing agrees with the MMSE timing phase for this example.

The performance of DM timing phase has not been investigated for the optical communication systems where channel is nonlinear and the noise is not Gaussian. In the following section we investigate the deviation of MSE performance of DM timing phase from the MMSE under various scenarios and OSNR.

\section{SIMULATION RESULTS}

In this section, we simulate optical transmission systems impaired by CD and PMD to find the optimal timing phase. The transmitter consists of $10 \mathrm{~Gb} / \mathrm{s}$ NRZ-OOK modulation. A CD/PMD emulator generates first order CD/PMD distortion with the CD dispersion parameter $D=10 \mathrm{ps} / \mathrm{km} / \mathrm{nm}$. The distorted signal is added with amplified spontaneous emission (ASE) noise. Using square-law detection, the electrical signal is obtained from the optical signal. We have used a 5th order Bessel low pass filter with $7 \mathrm{GHz}$ bandwidth after the conversion. The received signals are sampled with respect to two timing phases, one is MMSE timing and the other one is DM timing. For each channel and OSNR, we compare the MSE performance of the two timings and show their resulting channel estimates.

Figures 5 (a) and (b) show the performance of DM and MMSE timing phase (they both agree) for $80 \mathrm{~km}$ SMF with 10psec differential group delay under no noise and $30 \mathrm{~dB}$ OSNR. Figures 5 (c) and (d) also show that the performance of DM and MMSE timing phase (they both agree) for $D=80 \mathrm{~km}$ SMF with $\tau=60 \mathrm{psec}$ differential group delay under no noise and $30 \mathrm{~dB}$ OSNR. In these two examples, DM timing phase and MMSE timing phase agree in optical communication systems, and, therefore, the resulting baud-spaced ISI channels (each tap corresponds to $0.1 \mathrm{~ns}$ delay) agree as well.

\section{DISCUSSION AND CONCLUSION}

In this paper, we have shown that timing phase plays an important role for the electronic domain equalizers to mitigate $\mathrm{CD} / \mathrm{PMD}$ distortion. We proposed a blind timing phase optimization scheme based on dispersion minimization, called DM timing. The DM timing recovery algorithm achieves approximated MMSE 

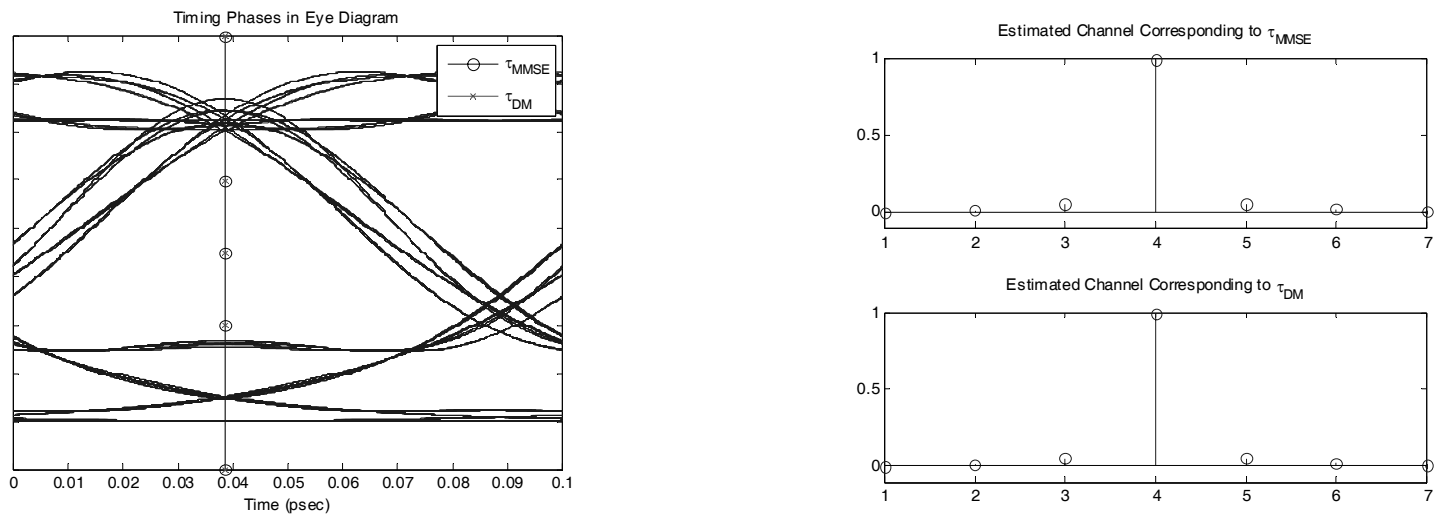

(a) MMSE and DM timing for $L=80 \mathrm{~km}$ and $\tau=10 \mathrm{ps}$ under Inf $\mathrm{dB}$ OSNR
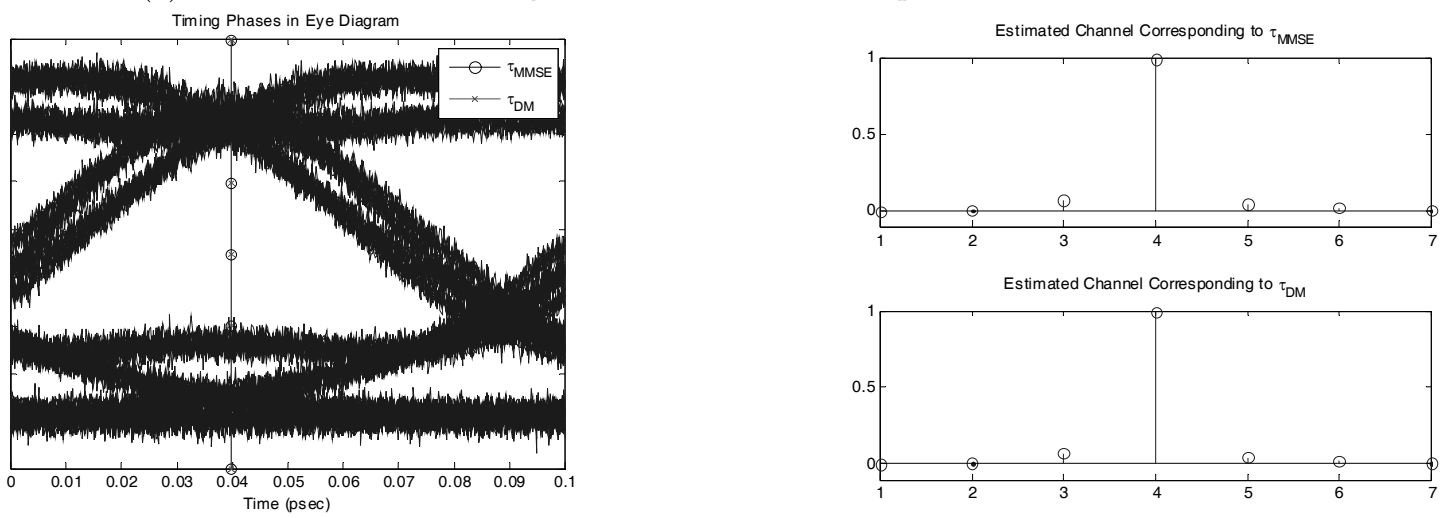

(b) MMSE and DM timing for $L=80 \mathrm{~km}$ and $\tau=10 \mathrm{ps}$ under $30 \mathrm{~dB}$ OSNR
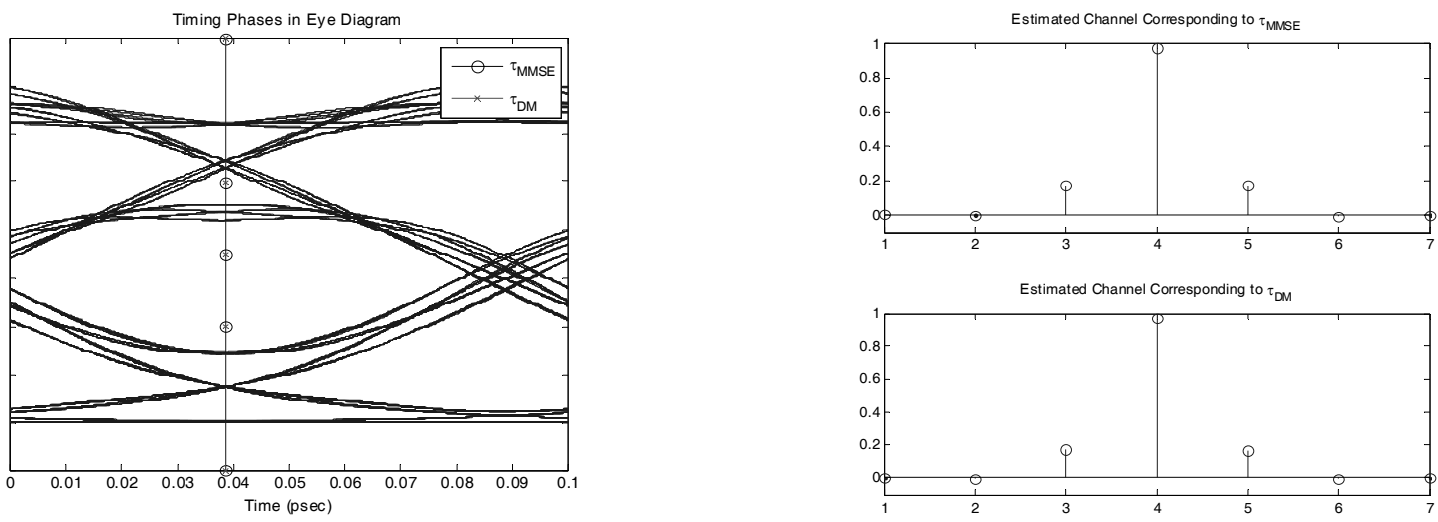

(c) MMSE and DM timing for $L=80 \mathrm{~km}$ and $\tau=60 \mathrm{ps}$ under Inf $\mathrm{dB}$ OSNR Timing Phases in Eye Diagram

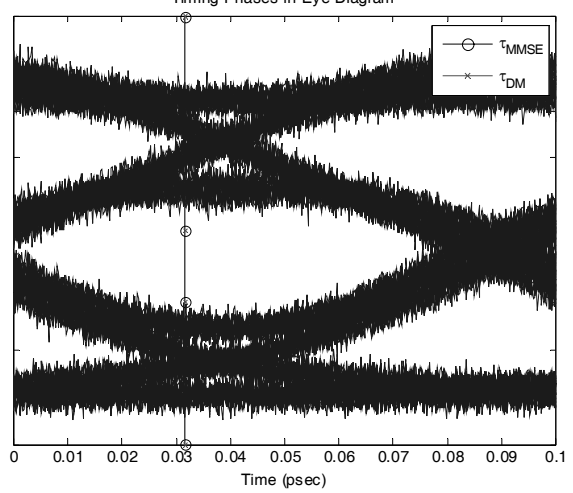

Estimated Channel Corresponding to $\tau_{\text {MMSE }}$

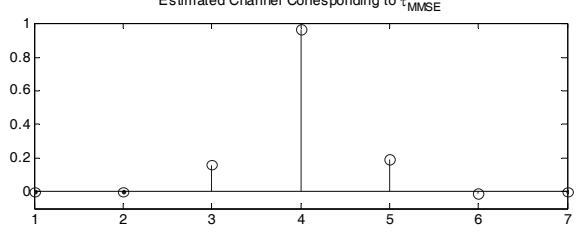

Estimated Channel Corresponding to $\tau_{\text {DM }}$

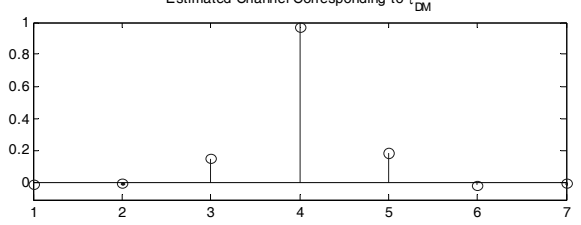

(d) MMSE and DM timing for $L=80 \mathrm{~km}$ and $\tau=60 \mathrm{ps}$ under $30 \mathrm{~dB}$ OSNR

FIG. 5. MMSE and DM timing for various distortion and OSNR. 
timing which is an optimal timing phase for equalization. Simulation results showed that the DM-timing agrees with the MMSE timing phase for several CD/ PMD environments.

\section{ACKNOWLEDGMENT}

This work was supported by the Brain Korea 21 project in 2009 .

\section{REFERENCES}

1. A. F. Elrefale, R. E. Wagner, D. A. Atlas, and D. G. Daut, "Chromatic dispersion limitations in coherent lightwave transmission systems," J. Lightwave Technol. 6, 704-709 (1988).

2. C. D. Poole and R. E. Wagner, "Phenomenological approach to polarization dispersion in long single-mode fibers," Electron. Lett. 22, 1029-1030 (1986).

3. H. Jang, K. Kim, J. Lee, and J. Jeong, "PMD tolerance of 10 Gbps modulated signals due to SOA-induced chirp in SOA booster amplifiers," J. Opt. Soc. Korea 12, 232-239 (2008).

4. S. J. Savory, "Digital filters for coherent optical receivers," Opt. Exp. 16, 804-817 (2008).

5. J. Wang and J. M. Kahn, "Performance of electrical equalizers in optically amplified OOK and DPSK systems," IEEE Photon. Technol. Lett. 16, 1397-1399 (2004).

6. W. Chen, F. Buchali, X. Yi, W. Shieh, J. S. Evans, and R. S. Tucker, "Chromatic dispersion and PMD miti- gation at $10 \mathrm{~Gb} / \mathrm{s}$ using Viterbi equalization for DPSK and DQPSK modulation formats," Opt. Exp. 15, 5271-5276 (2007).

7. C. Xia and W. Rosenkranz, "Nonlinear electrical equalization for different modulation formats with optical filtering," J. Lightwave Technol. 25, 996-1001 (2007).

8. W. Rosenkranz and C. Xia, "Electrical equalization for advanced optical communication systems," International Journal of Electronics and Communications 61, 153-157 (2007).

9. Y. Benlachtar, R. I. Killey, and P. Bayvel, "The effects of polarization-mode dispersion on the phase of the recovered clock," J. Lightwave Technol. 24, 3944-3952 (2006).

10. O. Coskun and K. M. Chugg, "Baud rate timing recovery and slicer threshold estimation for the adaptive dispersion compensation of fiber optical channels," in Proc. Thirty-seventh Asilomar Conference on Signals, Systems E3 Computers (Asilomar, CA, USA, 2003), vol. 1, pp. 27-31.

11. J. Proakis, Digital Communication Systems (McGrawHill Science, New York, USA, 2000).

12. W. Chung, "Timing phase offset recovery based on dispersion minimization," IEEE Trans. Signal Processing 53, 1097-1109 (2005).

13. K. S. Kim, J. Lee, W. Chung, and S. C. Kim, "An electronic domain chromatic dispersion monitoring scheme insensitive to osnr using kurtosis," J. Opt. Soc. Korea 12, 249-254 (2008).

14. J. R. Treichler, C. R. Johnson, and M. G. Larimore, Theory and Design of Adaptive Filters (Prentice Hall, Englewood Cliffs, NJ, USA, 2001). 